\title{
Not much helicity is needed to drive large-scale dynamos
}

\author{
Jonathan Pietarila Graham, ${ }^{1}$ Eric G. Blackman, ${ }^{2}$ Pablo D. Mininni, ${ }^{3,4}$ and Annick Pouquet ${ }^{3}$ \\ ${ }^{1}$ Solid Mechanics and Fluid Dynamics (T-3) and Center for Nonlinear Studies, Los Alamos National Laboratory MS-B258, \\ Los Alamos, New Mexico 87545, USA \\ ${ }^{2}$ Department of Physics and Astronomy, University of Rochester, Rochester, New York 14627, USA \\ ${ }^{3}$ Computational and Information Systems Laboratory, National Center for Atmospheric Research, P.O. Box 3000, Boulder, \\ Colorado 80307-3000, USA \\ ${ }^{4}$ Departamento de Física, Facultad de Ciencias Exactas y Naturales, Universidad de Buenos Aires and Instituto de Física de Buenos Aires \\ (IFIBA), Consejo Nacional de Investigaciones Científicas y Técnicas, Ciudad Universitaria, 1428 Buenos Aires, Argentina
}

(Received 15 August 2011; revised manuscript received 23 April 2012; published 20 June 2012)

\begin{abstract}
Understanding the in situ amplification of large-scale magnetic fields in turbulent astrophysical rotators has been a core subject of dynamo theory. When turbulent velocities are helical, large-scale dynamos that substantially amplify fields on scales that exceed the turbulent forcing scale arise, but the minimum sufficient fractional kinetic helicity $f_{h, C}$ has not been previously well quantified. Using direct numerical simulations for a simple helical dynamo, we show that $f_{h, C}$ decreases as the ratio of forcing to large-scale wave numbers $k_{F} / k_{\min }$ increases. From the condition that a large-scale helical dynamo must overcome the back reaction from any nonhelical field on the large scales, we develop a theory that can explain the simulations. For $k_{F} / k_{\min } \geqslant 8$ we find $f_{h, C} \lesssim 3 \%$, implying that very small helicity fractions strongly influence magnetic spectra for even moderate-scale separation.
\end{abstract}

DOI: 10.1103/PhysRevE.85.066406

PACS number(s): 52.30.Cv, 47.65.Md, 95.30.Qd

\section{INTRODUCTION}

The origin of magnetic fields in turbulent astrophysical rotators such as stars, galaxies [1], and accretion disks has been a longstanding topic of research. A particular challenge has been to understand the origin of fields on scales that are large compared to those of any underlying turbulence [1-4].

That the large-scale field of the sun reverses every 11 years reveals that such stellar fields cannot be simply the residual of flux freezing from the primordial material and must be amplified in situ. Complementarily, the continuous processing by supernovae-driven turbulence in galaxies likely renders the role of any primordial fields to be simply seed fields whose in situ processing must be understood to account for the observed present-day large-scale fields in galaxies. The presence of astrophysical jets from accretion engines also highlights the presence of large-scale fields in accretion disks, and accretion disk simulations [3] commonly show the in situ generation of large-scale magnetic fields that reverse on cycle periods of tens of orbit times.

The study of in situ field amplification in the presence of velocity flows is the enterprise of dynamo theory. Smallscale dynamos (SSDs), in which turbulent velocity flows amplify fields at or below scales of the forcing $[5,6]$, can be distinguished from large-scale dynamos (LSDs) in which magnetic fields are amplified on spatial or temporal variation scales larger than the scales of the underlying forcing. LSDs and SSDs are often contemporaneous and interactive (see, e.g., Refs. [4,7]) but LSDs arise only when turbulent velocities are sufficiently helical $[8,9]$. There has been little previous work, however, on determining the minimum sufficient helicity to incite LSD action and this is the topic of the present paper. Astrophysical flows are unlikely to be $100 \%$ helical in environments where LSDs are presumed; the galaxy for example is estimated to have helicity of $<10 \%$. Thus the basic question of how much helicity is required in even the simplest LSDs is important in assessing the potential ubiquity of LSDs.
The standard twentieth century textbook [10] kinematic approach to LSD theory has been classical mean-field theory (MFT), which features the $\alpha$ effect: $\gamma=|\alpha| k-\beta k^{2}$, where $\gamma$ is the exponential growth rate in the kinematic dynamo regime (presuming that any Lorentz-force feedback is negligible), $k$ is the wave number of magnetic field growth, the $\alpha$ effect is proportional to kinetic helicity $H_{v}=\langle\mathbf{v} \cdot \boldsymbol{\omega}\rangle$, with $\mathbf{v}$ the velocity and $\omega=\nabla \times \mathbf{v}$ the vorticity, and $\beta$ is the turbulent eddy diffusivity [10]. Such mean-field theory has been used to model solar [11], stellar, and galactic observations, as well as laboratory plasma dynamos [12], and geodynamos [13].

But the kinematic approach to LSD theory is incomplete. Although many astrophysical rotators have differential rotation and open boundaries, substantial progress in going beyond the kinematic theory has emerged from studies of the closed volume $\alpha^{2}$ helical dynamo without shear, in which the evolution of an initially weak seed field is subject to helical velocity forcing. The $\alpha^{2}$ dynamo was first tackled semianalytically [9] using a spectral integro-differential model with an eddy-damped quasinormal Markovian (EDQNM) closure, consistently tracking the magnetic helicity. It was shown that the actual driver of large-scale magnetic field growth is not just the kinetic helicity, but the residual helicity, $H_{R}=H_{v}-H_{j}$ where the current helicity $H_{j}=\langle\mathbf{j} \cdot \mathbf{b}\rangle$ and $\mathbf{j}=\nabla \times \mathbf{b}$ is the current density.

This $\alpha^{2}$ dynamo in a periodic box was simulated [14] by forcing with kinetic helicity at wave number $k_{F}=5 k_{\min }$ $\left(k_{\min }=1\right.$ was the smallest wave number of the flow). The large-scale $(k<5)$ field grew as expected from Ref. [9]. Subsequently, a two-scale $\alpha^{2}$ LSD was developed [15]; it incorporated magnetic helicity evolution using a simpler closure than EDQNM and showed even a two-scale nonlinear theory predicts the evolution and saturation of LSD growth observed in Ref. [14]. Driving with kinetic helicity initially produces a large-scale helical magnetic field, but the near conservation of magnetic helicity leads to a compensating small-scale magnetic (and current) helicity of opposite sign. 
This counteracts the kinetic helicity driving in the large-scale field growth coefficient, and quenches the LSD, as proposed in Ref. [9].

There has also been a plethora of work on the SSD. In a periodic box with a weak initial seed field and nonhelical forcing, the stochastic line stretching produces negligible field growth above the forcing scale. Simulations of nonhelical SSDs without large-scale shear show that the total magnetic energy is amplified to near equipartition with the total kinetic energy not only in the kinematic regime as predicted by Ref. [5], but also in the saturated regime for large magnetic Prandtl number $P_{M}=v / \eta$, where $v$ and $\eta$ are the viscosity and magnetic diffusivity [6,16,17], as reviewed in Ref. [7]. Astrophysical plasmas such as the galactic interstellar medium do not seem to exhibit this pileup [18].

To address this disparity between simulations of nonhelical SSDs and how conditions favorable for LSD growth might influence the magnetic spectrum on both large and small scales, results for dynamos in a periodic box forced with different amounts of fractional kinetic helicity $f_{h}$ (a dimensionless measure of the degree of alignment between the velocity and the vorticity of the forcing function), were studied [19]. It was found that the magnetic spectrum above and below the forcing scale were contemporaneously affected by a sufficient $f_{h}$. The large-scale field grew, and the magnetic spectrum at large wave numbers steepened. For $f_{h}=1$ and $f_{h}=0$, the results of Refs. [14,17] were respectively recovered.

But the restriction in Ref. [19] to a forcing scale of $k_{F}=5$ and resolution of $64^{3}$ grid points left key unexplored questions. In particular, the minimum $f_{h}$ for LSD action, $f_{h, C}$, could not be determined as a function of $k_{F}$. The smaller this minimum, the potentially more ubiquitous LSD conditions are in astrophysics. Here we perform much higher-resolution simulations for fractionally helical dynamos and quantify how $f_{h, C}$ depends on $k_{F} / k_{\min }$. We also develop a theory that correctly predicts the dependence, seen in the simulations.

\section{EQUATIONS AND SETUP}

The incompressible MHD equations for velocity $\mathbf{v}$ and magnetic field $\mathbf{b}$ are

$$
\begin{aligned}
\partial_{t} \mathbf{v}+\omega \times \mathbf{v} & =\mathbf{j} \times \mathbf{b}-\nabla p+v \nabla^{2} \mathbf{v}+\mathbf{F} \\
\partial_{t} \mathbf{A} & =\mathbf{v} \times \mathbf{b}-\nabla \phi+\eta \nabla^{2} \mathbf{A} \\
\nabla \cdot \mathbf{v} & =0, \quad \boldsymbol{\nabla} \cdot \mathbf{A}=0 .
\end{aligned}
$$

The total pressure divided by the constant (unit) density $p$ and the potential $\phi$ are obtained self-consistently to ensure incompressibility and the Coulomb gauge. The Reynolds number is $\operatorname{Re}=U_{\mathrm{rms}} L_{0} / v$, with $U_{\mathrm{rms}}$ and $L_{0}=2 \pi \int E(k) k^{-1} d k /$ $\int E(k) d k$ the r.m.s. velocity and the integral scale respectively; the magnetic Reynolds number is defined as $R_{M}=U_{\mathrm{rms}} L_{0} / \eta$. In the following, $E$ denotes the total energy, and $E_{v}$ and $E_{b}$ denote the kinetic and magnetic energy respectively.

We employ a well-tested pseudospectral code that uses a hybrid parallelization, combining message passing interface (MPI) and OpenMP [20]. The computational box has size $[2 \pi]^{3}$, and wave numbers vary from $k_{\min }=1$ to $k_{\max }=N / 3$ using a standard $2 / 3$ de-aliasing rule, where $N$ is the number of grid points per direction.
The forcing applied at $k_{F}$ is $\mathbf{F} \equiv \mathbf{F}_{R}+c \mathbf{F}_{A} ; \mathbf{F}_{A}$ is an $\mathrm{ABC}$ flow at $k_{F}$, and $\mathbf{F}_{R}$ is the sum of all harmonic modes with $k=k_{F}$ and random phases. We choose $c$ for a given fractional helicity of $\mathbf{F},\left|f_{h}\right| \leqslant 1$, with $f_{h} \equiv\left\langle\mathbf{F} \cdot \boldsymbol{\omega}_{F}\right\rangle\left(\left\langle|\mathbf{F}|^{2}\right\rangle\left\langle\left|\boldsymbol{\omega}_{F}\right|^{2}\right\rangle\right)^{-1 / 2}$, where $\omega_{F}=\nabla \times \mathbf{F}$. The entire forcing has random phases applied, with a correlation time $t_{\text {cor }}=0.1$. In practice, the ratio of helical to nonhelical forcing magnitudes is $c \simeq$ $\left[f_{h} /\left(1-f_{h}\right)\right]^{1 / 2} \equiv R_{h}$. The kinetic helicity at $k_{F}$ is typically within $25 \%$ of $f_{h}$. By choosing dimensional length and time constants $l_{0} \propto k_{F}$ and $t_{0}$ (fixed), varying $k_{F}$ in our simulations corresponds to dimensionalized physical systems described by Eqs. (1), where the forcing scale is constant and the system size increases $\propto k_{F}$. The dimensionless velocity $v_{\text {rms }}$ and forcing are $\propto k_{F}^{-1}$, and the diffusivity $\propto k_{F}^{-2}$.

A hydrodynamic state is evolved for five forcing-scale eddy turnover times, $\tau$, before a magnetic seed field at $k=k_{\text {seed }}$ is introduced. In the hydro steady state, the resulting $\tau=2 \pi\left[k_{F} U_{\mathrm{rms}}\right]^{-1} \approx 4$.2. In all simulations, dimensionalized viscosity is constant, $k_{F}^{2} v=2.412 \times 10^{-2}$, and, arbitrarily, $P_{M}=4$ so that $P_{M}>1$ while limiting the computational cost (see Table I for further details).

TABLE I. Parameters: Runs are labeled by the forcing wave number $k_{F}$ followed by the percentage of helicity in the forcing $f_{h}$; $R_{M}$ and $k_{\text {seed }}$ are defined in the text $\left(\operatorname{Re}=R_{M} / 4\right)$. The SSD growth rate is $\gamma_{\mathrm{SSD}}$. $E_{b}^{s}$ is the magnetic energy and $H_{b}$ is the magnetic helicity, both at later times, between $60 \tau$ and $130 \tau$; the " $f$ " is for fluctuating, and " $g$ " is for growing exponentially (indicating a helical dynamo). Forcing wave numbers are $k_{F}=2,3,4,5,6$, and 8 for runs on grids of $192^{3}, 256^{3}, 384^{3}, 432^{3}, 512^{3}$, and $768^{3}$ points respectively.

\begin{tabular}{ccccccc}
\hline \hline Run & $R_{M}$ & $k_{\text {seed }}$ & $\gamma_{\mathrm{SSD}}$ & \multicolumn{1}{c}{$\gamma_{k=1}$} & $E_{b}^{s}$ & $-100 H_{b}$ \\
\hline $2-80$ & 1500 & {$[6.7,10.7]$} & 0.24 & $(-1.2 \pm 10) 10^{-4}$ & 0.2 & $0.5 f$ \\
$2-85$ & 1600 & - & 0.22 & $(5.6 \pm 0.7) 10^{-3}$ & 0.4 & $6 g$ \\
$2-90$ & 1600 & - & 0.24 & $(6.0 \pm 0.7) 10^{-3}$ & 0.4 & $8 g$ \\
$3-40$ & 2000 & {$[10,16]$} & 0.31 & $(-1.0 \pm 7.6) 10^{-4}$ & 0.1 & $0.1 f$ \\
$3-60$ & 1900 & - & 0.32 & $(3.7 \pm 7.3) 10^{-4}$ & 0.1 & $0.2 f$ \\
$3-69$ & 1700 & - & 0.27 & $(6.0 \pm 0.7) 10^{-3}$ & 0.1 & $1.0 g$ \\
$3-80$ & 2000 & - & 0.33 & $(8.6 \pm 1.4) 10^{-3}$ & 0.3 & $10 g^{\mathrm{a}}$ \\
$4-10$ & 1700 & {$[13.3,21.3]$} & 0.25 & $(-1.6 \pm 2.0) 10^{-3}$ & 0.04 & $0.008 f$ \\
$4-20$ & 1600 & - & 0.28 & $(5.9 \pm 0.6) 10^{-3}$ & 0.04 & $0.06 g$ \\
$4-40$ & 1600 & - & 0.25 & $(1.5 \pm 0.1) 10^{-2}$ & 0.06 & $0.3 g$ \\
$4-60$ & 1500 & - & 0.25 & $(2.8 \pm 0.2) 10^{-2}$ & 0.1 & $1.0 g$ \\
$4-80$ & 1600 & - & 0.27 & $(2.8 \pm 0.3) 10^{-2}$ & 0.1 & $1.9 g$ \\
$5-09$ & 2000 & {$[16.7,26.7]$} & 0.26 & $(5.9 \pm 4.5) 10^{-4}$ & 0.03 & $0.004 f$ \\
$5-19$ & 1900 & - & 0.26 & $(-2.5 \pm 0.7) 10^{-3}$ & 0.03 & $0.007 f$ \\
$5-40$ & 1800 & - & 0.27 & $(1.6 \pm 0.7) 10^{-3}$ & 0.03 & $0.03 g$ \\
$5-50$ & 1900 & - & 0.27 & $(3.5 \pm 1.4) 10^{-3}$ & 0.03 & $0.06 g$ \\
$5-60$ & 1800 & - & 0.28 & $(1.1 \pm 0.09) 10^{-2}$ & 0.04 & $0.3 g$ \\
$6-01$ & 1700 & {$[20,32]$} & 0.27 & $(2.2 \pm 11) 10^{-4}$ & 0.02 & $0.002 f$ \\
$6-05$ & 1700 & - & 0.24 & $(4.1 \pm 5.0) 10^{-4}$ & 0.02 & $0.003 g$ \\
$6-10$ & 1700 & - & 0.27 & $(-1.5 \pm 6.9) 10^{-4}$ & 0.02 & $0.004 g$ \\
$6-15$ & 1600 & - & 0.23 & $(1.1 \pm 0.7) 10^{-3}$ & 0.02 & $0.007 g$ \\
$6-20$ & 1700 & - & 0.27 & $(4.5 \pm 2.0) 10^{-3}$ & 0.03 & $0.01 g$ \\
$6-30$ & 1600 & - & 0.23 & $(3.6 \pm 0.9) 10^{-3}$ & 0.03 & $0.02 g$ \\
$6-40$ & 1600 & - & 0.23 & $(7.4 \pm 0.7) 10^{-3}$ & 0.03 & $0.06 g$ \\
$8-03$ & 1200 & {$[26.7,42.7]$} & 0.20 & $(5.4 \pm 1.9) 10^{-3}$ & 0.008 & $4 \cdot 10^{-4} g$ \\
\hline \hline
\end{tabular}

${ }^{\text {aRun 3-80 was pursued until } t \approx 300 \tau \text {, at which time } E_{b} \sim 0.3 \text { and }}$ $H_{b} \sim 10$. See Fig. 2. 


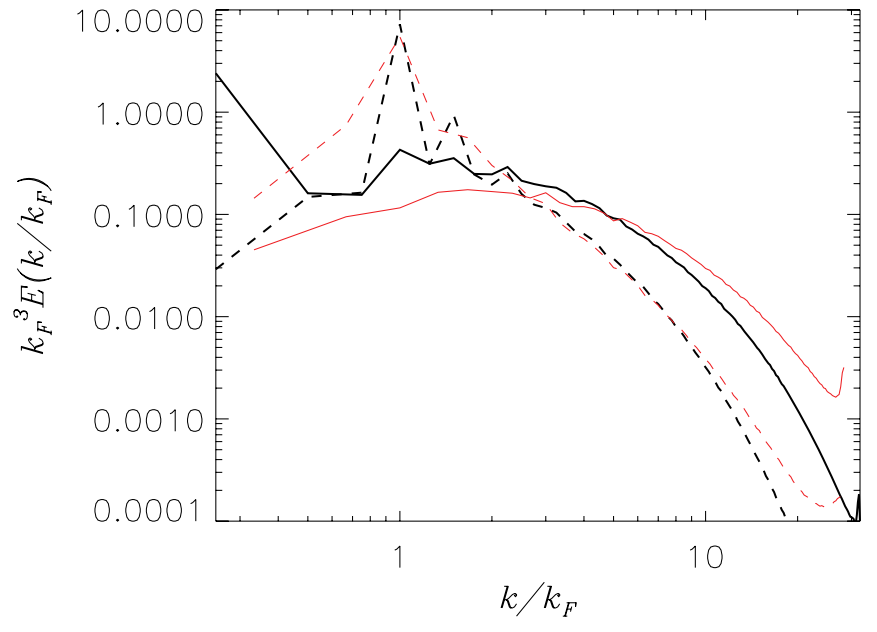

FIG. 1. (Color online) Redimensionalized magnetic (solid) and kinetic (dashed) energy spectra after $90 \tau$ for run 4-60 (thick black) forced at $k_{F}=4$ and for run 3-60 (thin red/light gray) with $k_{F}=3$. At a fixed $f_{h}=60 \%$ here, increased scale separation provides for the transition between SSD and LSD.

\section{SIMULATION RESULTS}

Table I summarizes our runs. The kinetic and magnetic energy spectra after $t=90 \tau$ are displayed in Fig. 1, for runs 4-60 (with $k_{F}=4$ ) and 3-60 (with $k_{F}=3$ ). Both have $f_{h}=$ $60 \%$, and for both, the small-scale fields grow; only for $k_{F}=4$ does $k=1$ grow.

Figure 2 shows the growth of magnetic energy in the $k=1$, $k=6$, and total over all modes for run 3-80. The evolution exhibits an early phase in which both modes grow at the same rapid rate, with $\gamma_{\mathrm{SSD}} \sim 0.33 \sim \tau^{-1}$, followed by a slow growth of the $k=1$ mode and a saturation for the $k=6$ mode. The $k=1$ mode accounts for nearly $10 \%$ of $E_{b}$ by $100 \tau$ and the growth rate slows, but has not fully saturated by $300 \tau$. The growth rate of magnetic energy at $k=1$ during the SSD phase is nearly the same for all of our runs, and is insensitive to $f_{h}$,

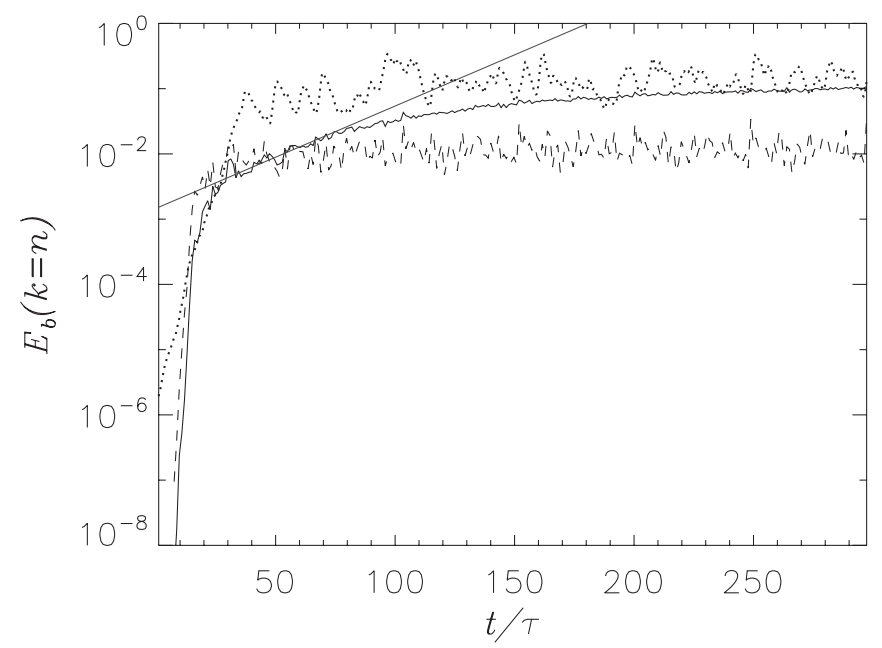

FIG. 2. Magnetic energy density $E_{b}(k)$ for $k=1$ (solid line), $k=6$ (dashed), and total (dotted) versus time for run 3-80. The gray line indicates the fit $\gamma=(8.6 \pm 1.4) 10^{-3}$ to the solid curve for growth of the $k=1$ mode after SSD saturation.

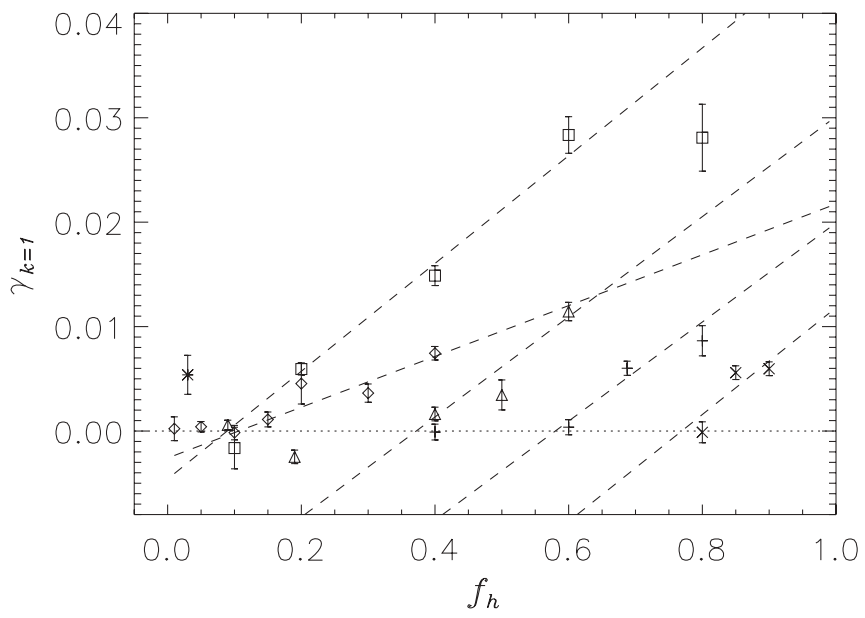

FIG. 3. Growth rate $\gamma_{k=1}$ of $E_{b}(k=1)$ versus fractional helicity. From Table I, $\times\left(k_{F}=2\right),+\left(k_{F}=3\right), \square\left(k_{F}=4\right), \triangle\left(k_{F}=5\right), \diamond\left(k_{F}=\right.$ $6), *\left(k_{F}=8\right)$, and least-squares linear fits (dashed).

$R_{M}$, and $k_{F}$. Sensitivity to $f_{h}$ emerges once the SSD regime ends.

The $\gamma_{k=1}$ growth rates [for $E_{b}(k=1)$ ] that immediately follow the SSD phase (see Table I) are shown in Fig. 3. This LSD growth regime occurs only when $f_{h}>f_{h, C}$; the LSD growth rate varies linearly with $f_{h}$ for a fixed $k_{F} / k_{\min }$ $\left(\alpha \propto H_{v} \propto f_{h}\right)$. Least-squares fits are dashed lines in Fig. 3 [the $y$ intercept, $\beta \sim\left(k_{\min } / k_{F}\right)^{2.2}$ ]. The short exponential growth phase of 4-80 makes for an inaccurate measure of $\gamma_{k=1}$; it is thus excluded from the fit. As $k_{F} / k_{\min }$ increases, $f_{h, C}$ decreases.

The LSD exponential growth of $E_{b}(k=1)$ for $f_{h} \geqslant f_{h, C}$ is accompanied by a $k=1$ growth of magnetic helicity. Studies of the $k=1$ growth for $f_{h}=1$ in a two-scale approach [14,15] for a $H_{R}$ driven dynamo [9] suggest two phases of $k_{\text {min }}=1$ mode growth after the SSD regime: one phase that is largely independent of $R_{M}$, and a subsequent $R_{M}$-dependent asymptotic regime. The former phase has growth consistent with our $\gamma_{k=1}$ phase. In all these runs, $f_{h, C}$ decreases with increasing $k_{F} / k_{\min }$, as displayed in Fig. 4. For the largest

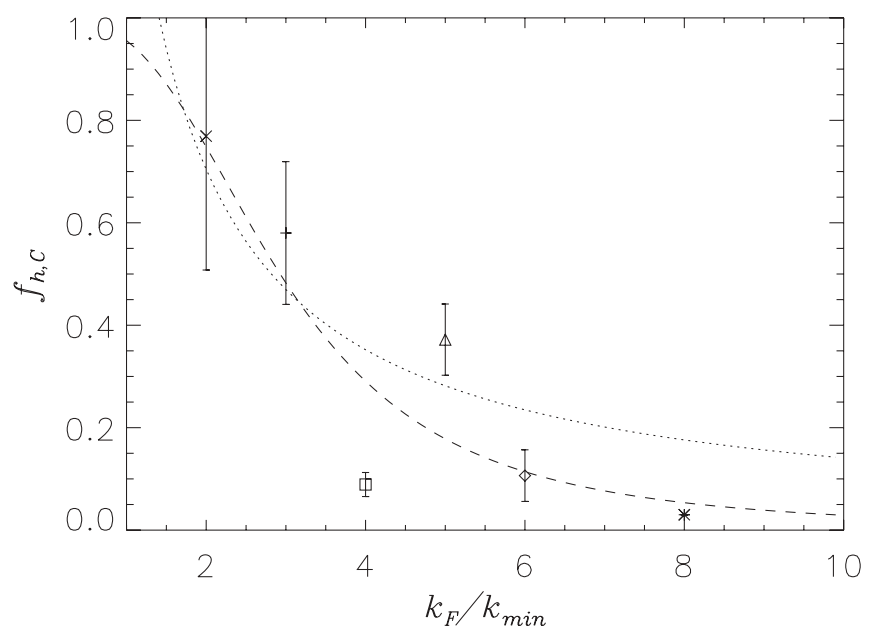

FIG. 4. $f_{h, C}$ from least-squares fits versus $k_{F} / k_{\min }$ (symbols as in Fig. 3). Dashed line is best fit to Eq. (2), giving $C=0.21$ and $\xi=$ 0.46. Dotted line is kinematic MFT prediction $f_{h, C}=\beta k_{\min } /\left(\left|\alpha_{0}\right| k_{F}\right)$. 
$k_{F} / k_{\min }(=8)$ case, $f_{h} \sim 3 \%$ is sufficient. In Fig. 4 , the error bars and $f_{h, C}$ are calculated as follows: The $x$ intercept from the least-squares linear fits, $\gamma_{k=1}=m f_{h}+b$ shown in Fig. 3, determine our estimate of $f_{h, C}=-b / m$ for Fig. 4 . The $1-\sigma$ uncertainties for $b$ and $m$ are then propagated for $f_{h, C}: \sigma_{f_{h, C}}=b / m \sqrt{\left(\sigma_{b} / b\right)^{2}+\left(\sigma_{m} / m\right)^{2}}$.

\section{THEORETICAL PREDICTION FOR $f_{h, c}$}

The following prediction for $f_{h, C}$ is based on the principle that LSD helical field growth at $k=k_{\text {min }}$ beyond the SSD phase requires helical velocity forcing to overcome the Lorentz force at $k=k_{\min }$ at the end of the kinematic SSD phase. For that time, we assume the magnetic energy at $k_{\min }<k_{F}$ to be $B_{\min }^{2} \sim B_{F}^{2}\left(k_{\min } / k_{F}\right)^{\xi}$, where $B_{F}$ is the magnetic field at $k_{F}$, and $\xi-1$ is the slope of the magnetic energy spectrum on a log-log plot. The associated Lorentz force is then $M_{n h}\left(f_{h}\right) B_{F}^{2} k_{F}\left(k_{\min } / k_{F}\right)^{\xi+1}$, where the function $M_{n h}\left(f_{h}\right)<1$ accounts for the contribution from only nonhelical magnetic energy.

The available helical velocity forcing that must overcome this Lorentz force is only a fraction of the helical forcing at $k=$ $k_{F}$ : At early times when magnetic helicity is nearly conserved, the forcing not only sources magnetic helicity at $k=k_{\min }$ but also an oppositely signed, equal in magnitude, magnetic helicity at $k=k_{s s} \geqslant k_{F}$. The associated ratio of helical magnetic energy growth at $k_{\min }$ to that at $k_{s s}$ is then $\sim k_{\min } / k_{s s}<1$. The helical force that needs to exceed the Lorentz force at $k_{\text {min }}$ to initiate growth is thus $\sim K_{h}\left(f_{h}\right) v_{\mathrm{rms}}^{2} k_{F}\left(k_{\min } / k_{s s}\right)$, where the function $K_{h}\left(f_{h}\right)<1$ accounts for only kinetic helical forcing.

Balancing the aforementioned forces assuming $K_{h} / M_{n h}=$ $R_{h}\left(f_{h}\right)$ (which is consistent with our data), and assuming $f_{h}=$ $f_{h, C}$, then gives

$$
f_{h, C}=\frac{1}{1+C^{2}\left(k_{F} / k_{\min }\right)^{2 \xi+2}},
$$

where $C \equiv\left(k_{\min } v_{\mathrm{rms}}^{2}\right) /\left(k_{s s} B_{F}^{2}\right) \sim k_{\min } / k_{s s}$. Figure 4 shows the data and the best fit using Eq. (2); $\xi=0.46 \approx 1 / 2$ is found. This yields the prediction, $f_{h, C} \sim\left(k_{F} / k_{\min }\right)^{-3}$ as $k_{F} / k_{\min } \rightarrow$ $\infty$. Note that taking the limit of infinite scale separation, we have a LSD with zero helicity (but only fluctuations, as in Ref. [21]).

The theory above, which considers the Lorentz force back reaction from the large-scale field, can be contrasted with the prediction from the purely kinematic theory of the standard $\alpha^{2}$ dynamo, which does not include any Lorentz forces. Using the formula presented in the introduction for the growth rate at $k_{\text {min }}$, and the definition of $f_{h}$, the critical fractional helicity for the kinematic theory would be $f_{h, C}=\beta k_{\min } /\left(\left|\alpha_{0}\right| k_{F}\right)$ where $\alpha_{0} \equiv \alpha / f_{h}$. This formula is shown as the dotted line in Fig. 4 and does not fit the data very well, highlighting the importance of including the Lorentz force. This does not imply the kinematic theory is irrelevant however. For values of $f_{h} \gg f_{h, C}$ the kinematic theory should be applicable to estimating the early time growth rate because the driving helicity overwhelms the back reaction associated with the weak large-scale field produced by the SSD in that regime.

\section{DISCUSSION OF LARGE-SCALE DYNAMOS GROWTH AND SATURATION}

At large $R_{M}$, SSD action produces field at all scales, potentially precluding a scale separation between the mean magnetic field and velocity fluctuations (an essential assumption to derive $\alpha^{2}$ MFT) [22]. The SSD magnetic energy spectrum at scales above the forcing scale produces less magnetic energy the larger the scale [5]. At large enough scales, the magnetic energy production from the SSD will be negligible, and scale separation becomes a meaningful concept (see thin red/light gray, solid line in Fig. 1). This helps justify the mean-field approach to LSDs.

In the mean-field, two-scale approach, once the small-scale magnetic helicity has grown as a result of magnetic helicity conservation to be large enough such that the associated smallscale current helicity back-reacts on the driving kinetic helicity, the $\alpha^{2}$ dynamo eventually slows to $R_{M}$-dependent growth rates and ultimately saturates completely. Previous studies have typically focused on the $f_{h}=1$ case $[14,15]$. In this paper, we have not run enough simulations long enough to determine how strong the large-scale field gets before its evolution reaches the $R_{M}$-dependent regime. However, if cases with fractional helicity $f_{h, C}<f_{h}<1$ saturate by direct analogy to the $f_{h}=1$ cases studied in previous work, then the value of the largescale magnetic energy reached just before the $R_{M}$-dependent regime emerges would be expected to be simply proportional to an extra factor of $f_{h}$, namely $\bar{B}^{2} \sim f_{h}\left(k_{1} / k_{f}\right)\left\langle U_{\text {rms }}^{2}\right\rangle$. Similarly, for asymptotically saturated steady state at very late times, we would expect $\bar{B}^{2} \sim f_{h}\left(k_{f} / k_{1}\right)\left\langle U_{\text {rms }}^{2}\right\rangle$. Note that in the $f_{h}=1$ case, the latter similarity highlights the fact that that superequipartition field strengths (with respect to the total kinetic energy) are able to grow by the end of the nonlinear, saturated regime for fully helical $\alpha^{2}$ dynamo.

Note however that the $R_{M}$-dependent regimes of the $\alpha^{2}$ dynamo are largely irrelevant for astrophysical objects, which have such large $R_{M}$ that something else probably happens before these regimes are reached. Open boundaries and helicity fluxes are ingredients that have to be considered in realistic systems. In addition, real astrophysical dynamos have largescale shear, which amplifies the total large-scale field beyond its purely helical value. More work is needed to determine the strength of the large-scale fields produced by fractionally helical LSDs.

\section{CONCLUSION}

Only a minuscule amount of fractional helicity is required for LSD action at even modest astrophysically relevant scale separations. For $f_{h}>f_{h, C}$, the $k=1$ field grows and the small-scale spectrum steepens (see Fig. 1 and Ref. [19]). This may be important because our result that $f_{h, C} \lesssim 3 \%$ for $k_{F} / k_{\min } \geqslant 8$ offers a basic principle for potentially reconciling a disparity between a pileup of small-scale magnetic energy in large $P_{M}$ nonhelical dynamo simulations [6] and Galactic observations [18]. Our results also suggest that large-scale separations should be a priority in designing laboratory experiments to measure LSD action [2]. 


\section{ACKNOWLEDGMENTS}

Computer time was provided by the National Center for Atmospheric Research (NCAR), Los Alamos National Laboratory, and Max-Planck-Institut für Sonnensystemforschung.
NCAR is sponsored by NSF. P.D.M. was supported by grants PIP 11220090100825 and PICT-2007-02211. J.P.G. gratefully acknowledges the support of the US Department of Energy through the LANL/LDRD Program for this work.
[1] R. Beck et al., Annu. Rev. Astron. Astrophys. 34, 155 (1996).

[2] D. Lathrop and C. Forest, Phys. Today 64, 40 (2011).

[3] A. Brandenburg, Space Sci. Rev. 144, 87 (2009).

[4] E.G. Blackman, Astron. Nachr. 331, 101 (2010).

[5] A.P. Kazantsev, Sov. Phys. JETP 26, 1031 (1968).

[6] A.A. Schekochihin et al., Astrophys. J. 612, 276 (2004)

[7] A. Brandenburg and K. Subramanian, Phys. Rep. 417, 1 (2005).

[8] M. Meneguzzi, U. Frisch, and A. Pouquet, Phys. Rev. Lett. 47, 1060 (1981).

[9] A. Pouquet, U. Frisch, and J. Léorat, J. Fluid Mech. 77, 321 (1976).

[10] H. Moffatt, Magnetic field generation in electrically conducting fluids (Cambridge University Press, Cambridge, 1978).

[11] A. Brun, M. Miesch, and J. Toomre, Astrophys. J. 614, 1073 (2004); M. Browning, ibid. 676, 1262 (2008).

[12] A. Gailitis et al., Rev. Mod. Phys. 74, 973 (2002); H. Ji and S. Prager, Magnetohydrodynamics 38, 191 (2002).
[13] P. Roberts and G. Glatzmaier, Rev. Mod. Phys. 72, 1081 (2000); F. Busse, Annu. Rev. Fluid Mech. 32, 383 (2000).

[14] A. Brandenburg, Astrophys. J. 550, 824 (2001).

[15] E. G. Blackman and G. B. Field, Phys. Rev. Lett. 89, 265007 (2002).

[16] N. E. L. Haugen, A. Brandenburg, and W. Dobler, Astrophys. J. Lett. 597, L141 (2003); Phys. Rev. E 70, 016308 (2004).

[17] A. A. Schekochihin et al., Astrophys. J. 576, 806 (2002)

[18] A. H. Minter and S. R. Spangler, Astrophys. J. 458, 194 (1996)

[19] J. Maron and E. Blackman, Astrophys. J. Lett. 566, L41 (2002).

[20] P. D. Mininni et al., Parallel Computing 37, 316 (2011).

[21] A. Gilbert, U. Frisch, and A. Pouquet, Geophys. Astrophys. Fluid Dyn. 42, 151 (1988).

[22] F. Cattaneo and D. Hughes, Mon. Not. R. Astron. Soc. 395, L48 (2009). 